\title{
Association of dyskalemias with short-term health care utilization in patients with advanced CKD
}

\author{
Ankur A Dashputre, MS; Justin Gatwood, MPH, PhD; Keiichi Sumida, MD, MPH, PhD; Fridtjof Thomas, PhD; \\ Oguz Akbilgic, PhD; Praveen K Potukuchi, MS, PhD; Yoshitsugu Obi, MD, PhD; Miklos Z Molnar, MD, PhD; \\ Elani Streja, MPH, PhD; Kamyar Kalantar-Zadeh, MD, PhD; and Csaba P Kovesdy, MD
}

\section{What is already known about this subject}

- Dyskalemias (especially hyperkalemia) are common in patients with advanced chronic kidney disease (CKD) and associated with adverse outcome such as mortality, cardiovascular events, and higher health care burden.

- Information is scarce regarding the association of dyskalemias with shortterm hospital/emergency room (ER) visits in an advanced CKD population.

\section{What this study adds}

- Hyper- and hypokalemia are associated with a higher risk of short-term hospital and ER visits, with a higher risk associated with hyperkalemia.

- Study results shed further light on the short-term risk of hospital/ER visits due to dyskalemias.

- Correcting and especially preventing dyskalemias could reduce hospital/ ER visits and associated costs-close monitoring of $\mathrm{K}$ levels and the advent of newer $\mathrm{K}$ binding agents (in the context of hyperkalemia) might help in reducing health care utilization and eventual economic burden.

\author{
Author affiliations \\ Ankur A Dashputre, MS, Institute for \\ Health Outcomes and Policy, College of \\ Graduate Health Sciences, University of \\ Tennessee Health Science Center, Memphis. \\ Justin Gatwood, MPH, PhD, Department \\ of Clinical Pharmacy and Translational \\ Science, College of Pharmacy, University \\ of Tennessee Health Science Center, \\ Nashville. Fridtjof Thomas, PhD, Division \\ of Biostatistics, Department of Preventive \\ Medicine, College of Medicine, University \\ of Tennessee Health Science Center, \\ Memphis. Oguz Akbilgic, PhD, Department \\ of Health Informatics and Data Science, \\ Parkinson School of Health Sciences and \\ Public Health, Loyola University, Chicago, \\ IL. Keiichi Sumida, MD, MPH, PhD; Praveen \\ K Potukuchi, MS, PhD; and Yoshitsugu \\ Obi, MD, PhD, Division of Nephrology, \\ Department of Medicine, University of
}

\section{ABSTRACT}

BACKGROUND: Patients with advanced chronic kidney disease (CKD) are at high risk for dyskalemias, which may induce arrhythmias that require immediate emergent or hospital care. The association of dyskalemias with short-term hospital/emergency room (ER) visits in advanced CKD is understudied.

OBJECTIVE: To assess the association of dyskalemias with short-term hospital/ER visits in an advanced CKD population.

METHODS: From among 102,477 US veterans transitioning to dialysis from 2007 to 2015 , we identified 21,366 patients with 2 predialysis
Tennessee Health Science Center, Memphis. Miklos Z Molnar, MD, PhD, Division of Nephrology \& Hypertension, Department of Medicine, University of Utah, Salt Lake City. Elani Streja, MPH, PhD, and Kamyar Kalantar-Zadeh, MD, PhD, Harold Simmons Center for Chronic Disease Research and Epidemiology, Division of Nephrology and Hypertension, University of CaliforniaIrvine, Orange, CA. Csaba P Kovesdy, MD, Nephrology Section, Memphis VA Medical Center, Memphis, TN.

\section{AUTHOR CORRESPONDENCE: \\ Csaba P Kovesdy, 901.523.8990; \\ ckovesdy@uthsc.edu}

J Manag Care Spec Pharm 2021;27(10):1403-15

Copyright $(2021$, Academy of Managed Care Pharmacy. All rights reserved.

outpatient eGFR<30 ml/min/1.73m² 90-365 days apart (with the second eGFR serving as the index date) and at least 1 potassium (K) in the baseline period ( 1 year before index) and 1 outpatient $\mathrm{K}$ (oK) in the follow-up (1 year after the index but before dialysis initiation). We examined the association of time-varying hypokalemia $(\mathrm{K}<3.5 \mathrm{mEq} / \mathrm{L})$ and hyperkalemia $(K>5.5 \mathrm{mEq} / \mathrm{L})$ vs referent (3.5-5.5 mEq/L) with separate hospital and ER visits within 2 calendar days following each oK value over the 1-year follow-up period from the index. We used generalized estimating equations with binary distribution and logit link to model the exposureoutcome relationship adjusted for various 
confounders. We conducted various subgroup and sensitivity analyses to test the robustness of our results.

RESULTS: Over the 1-year follow-up, 125,266 oK measurements were observed, of which $6.8 \%$ and $3.7 \%$ were classified as hyper- and hypokalemia, respectively. In the multivariable-adjusted model, hyperkalemia (adjusted odds ratio $[\mathrm{aOR}]=2.04 ; 95 \% \mathrm{Cl}=1.88-2.21$ ) and hypokalemia $(\mathrm{aOR}=1.66 ; 95 \% \mathrm{Cl}=1.48-1.86)$ were associated with significantly higher odds of hospital visits. Similarly, hyperkalemia $(\mathrm{aOR}=1.83 ; 95 \% \mathrm{Cl}=1.65-2.03)$ and hypokalemia $(\mathrm{aOR}=1.24 ; 95 \%$ $\mathrm{Cl}=1.07-1.44)$ were associated with significantly higher odds of ER visits. Results were robust to subgroups and sensitivity analyses.

CONCLUSIONS: In patients with advanced CKD, dyskalemias are associated with higher risk of hospital/ER visits. Interventions targeted at lowering the risk of dyskalemias might help in reducing the health care utilization and associated economic burden among patients with advanced CKD experiencing dyskalemias.

Serum potassium $(\mathrm{K})$ is maintained within a narrow range of 3.5-5.0 milliequivalents per litre $(\mathrm{mEq} / \mathrm{L})$ by the kidneys, thus, patients with chronic kidney disease (CKD) are prone to dyskalemias (hypo- and hyperkalemia, especially the latter). ${ }^{1,2}$ The prevalence of hypo- and hyperkalemia in CKD (irrespective of CKD stage and depending on the K threshold) is between $12 \%-31 \%$ and $14 \%-41 \%$, respectively, with a higher prevalence among those with advanced CKD. ${ }^{3-8}$ In addition, patients with advanced CKD may be chronic users of dyskalemia-inducing medications such as renin-angiotensin-aldosterone system inhibitors (RAASi) and diuretics for comorbidity management (eg, cardiovascular diseases [CVD] $){ }^{3,9}$ Across various populations, dyskalemias are associated with adverse outcomes, including higher mortality, cardiovascular events, and health care burden., ${ }^{4,510-12}$

Dyskalemias induce arrhythmias, which may require immediate hospital/emergency room (ER) visits, and may also lead to sudden cardiac death., ${ }^{1,3}$ Previous research in CKD and hemodialysis populations suggests that dyskalemias are associated with higher risk of hospital and ER visits. ${ }^{5,11,13-16}$ Some of the previous studies assessed the association of hyperkalemia (using a single $\mathrm{K}$ value) with hospital/ER visits observed over 6-months or more following a measured $K$ value..$^{11,14-16}$ Others used repeated $K$ values and assessed outcomes over patient-period following each $\mathrm{K}$ value or within 4 days of $\mathrm{K}$ measurement.,13 While the studies using a single $\mathrm{K}$ value showed how a single dyskalemia event might affect future distant outcomes, ${ }^{11,14-16}$ the latter 2 studies, ${ }^{5,13}$ using repeated $\mathrm{K}$ values and short outcome assessments period, better mimic the transient nature of dyskalemia-associated events.
Patients with advanced CKD transitioning to dialysis represent a unique population with high comorbidity burden (eg, hypertension and CVD) and are in frequent need of pharmacotherapeutic interventions (eg, RAASi and diuretics). ${ }^{17}$ These patients are thus at a higher risk of dyskalemias (especially hyperkalemia) because of gradually declining kidney function, comorbidities, and use of medications affecting $\mathrm{K}$ levels and may experience higher rates of dyskalemia-associated hospital/ER visits. Although previous studies in CKD have described the association of dyskalemias with hospital/ER visits, they are either limited by use of single $\mathrm{K}$ value and long outcome assessment periods, ${ }^{11,14-16}$ use of patient-period (which might be long or short) following $\mathrm{K},{ }^{5}$ focused on hemodialysis patients, ${ }_{13}^{13}$ or focused mainly on hyperkalemia (and not hypokalemia). ${ }^{11,13-16}$ The aim of this study was to assess the association of dyskalemias with short-term hospital/ER visits in an advanced CKD population transitioning to dialysis.

\section{Methods}

\section{STUDY POPULATION}

A retrospective analysis of longitudinal data from the Transition of Care in Chronic Kidney Disease cohort, a nationally representative historical cohort of US veterans (sourced from the United States Renal Data System [USRDS]; $n=102,477)$ with incident end-stage renal disease (ESRD) transitioning to dialysis from October 1, 2007, through March 31, 2015, was conducted. ${ }^{18-22}$ An initial sample of 60,520 US veterans with predialysis outpatient estimated glomerular filtration rate (eGFR) data was identified. Among these, we identified 36,644 with 2 outpatient eGFR $<30 \mathrm{ml} /$ min $/ 1.73 \mathrm{~m}^{2}$ measured $90-365$ days apart, with the second eGFR serving as the index.

Further, the sample was restricted to 23,363 , with at least a 1-year look-back period (baseline) and follow-up period (before dialysis initiation) from the index for each. Among these, 21,602 had at least $1 \mathrm{~K}$ value in the baseline and 1 outpatient $\mathrm{K}(\mathrm{oK})$ value in the follow-up period.

Finally, we excluded 236 patients (aged $<18$ years at index $[\mathrm{n}=1]$ and without medication data $[\mathrm{n}=235])$ to yield a final sample size of 21,366 patients (Supplementary Figure 1, available in online article).

\section{EXPOSURE}

The exposure of interest was the time-varying oK value captured during the 1-year follow-up period from the index. Each time-varying oK value was categorized as hypokalemia $(\mathrm{K}<3.5 \mathrm{mEq} / \mathrm{L})$, hyperkalemia $(\mathrm{K}>5.5 \mathrm{mEq} / \mathrm{L})$, and referent $(3.5 \leq \mathrm{K} \leq 5.5 \mathrm{mEq} / \mathrm{L}){ }^{23}$ 


\section{COVARIATES}

Patient demographic characteristics were extracted from the USRDS Patient and Medical Evidence file. Data on marital and smoking status were obtained from VA records. ${ }^{24,25}$ Preexisting comorbidities as of the index were identified from the VA Inpatient and Outpatient Medical SAS, and the VA/Centers for Medicare \& Medicaid Services (CMS) databases, using a 2 outpatient or 1 inpatient claims algorithm of the International Classification of Diseases, Ninth Revision, Clinical Modification (ICD-9-CM) diagnostic and Current Procedural Terminology (CPT) codes. The Charlson Comorbidity Index (CCI) score was calculated using the Deyo modification for administrative datasets as a measure of comorbidity burden with kidney disease excluded from the algorithm. ${ }^{26}$

Data on prescribed medications were collected in the baseline period and as a time-varying covariate from VA pharmacy dispensation records and CMS Medicare Part D. For the baseline period, patients were considered to be users if they had at least one 30-day supply dispensation for chronic medications (RAASi, loop diuretics, K-sparing diuretics, thiazide diuretics, nonsteroidal anti-inflammatory drugs, digoxin, beta blockers, calcium channel blockers, insulin, oral hypoglycemics, beta-2 agonist, and calcineurin inhibitors) and at least 1 dispensation of any days supply for sodium polystyrene sulphonate (SPS), trimethoprim, azole antifungals, and laxatives.

Time-varying medications were captured at each oK during the follow-up period such that if the prescription date was the same as the oK measurement date or if the oK measurement fell within the days supply of the medication. Laboratory and vital signs data were captured over the baseline period and were obtained from VA research databases as previously described. ${ }^{27,28}$ The eGFR was calculated by the Chronic Kidney Disease Epidemiology Collaboration equation, using outpatient serum creatinine values. ${ }^{29}$ Outpatient eGFR was assessed at the index and as a time-varying covariate captured at each oK measurement over the 1-year follow-up.

\section{OUTCOMES}

The outcomes of interest were hospital visits and ER visits (treated as separate events) observed within 2 calendar days of each time-varying oK value captured during the 1-year follow-up period from the index. ER visits that were observed on the same day or on the day before a hospital visit were not counted as an ER visit, since they potentially could have led to the subsequent hospital visit. Reason for hospital visit was identified based on the primary diagnosis and categorized into "diagnosis chapters" as specified by the Agency for Healthcare Research and Quality (AHRQ) Clinical Classifications Software (CCS) for ICD-9-CM. ${ }^{30}$

\section{STATISTICAL ANALYSIS}

Patient characteristics were summarized for the entire sample and by hypokalemia, hyperkalemia, and referent categories (previously described) based on average $\mathrm{K}$ levels in the baseline. Data were presented as counts (percentages), mean (SD), or median (25th-75th percentiles), and differences across $\mathrm{K}$ categories were assessed using chi-square tests, 1-way analysis of variance, and Kruskal-Wallis tests, as appropriate. In the 1-year follow-up period, the proportion (95\% CI) of hospital and emergency visits over all the measured oK values was reported using binomial proportions. The association between categorized time-varying oK value (clustered within each individual patient) and hospital/ER visits within 2 calendar days of the K measurement was assessed using generalized estimating equations (GEE) with a binomial distribution, logit link, and independent correlation matrix.

Incrementally multivariable-adjusted models (models 1-6) accounting for confounders based on theoretical considerations and availability in the database were conducted. Supplementary Table 1 (available in online article) elaborates on the incrementally adjusted models (models 1-6) and the confounders accounted for in each model. Briefly, the models were conducted as follows-model 1: unadjusted; model 2: adjusted for sociodemographics; model 3: adjusted for smoking status, baseline averaged vital signs, history of comorbidities, CCI, and baseline health care utilization; model 4: adjusted for baseline medication use; model 5: index eGFR value, index year, baseline-averaged $\mathrm{K}$ levels and characteristics (number of K counts and hyper-/ hypokalemia events), and baseline-averaged bicarbonate levels; and model 6 (fully adjusted): time-varying eGFR and medications captured at each oK during the 1-year followup period.

We also modeled the relationship between categorized time-varying oK value and reason for hospital visit. We assessed the relationship between categorized time-varying oK and hospital visits categorized as "diseases of the circulatory system" (AHRQ CCS 96-121, event of interest); "other" (all other reasons as captured by AHRQ CCS); and no hospital visit (reference). In addition, we assessed the association between categorized time-varying oK value with hospital visits due to "arrhythmias" (AHRQ CCS 105-107). Similarly, we assessed the relationship between categorized time-varying oK value and hospital visits categorized as "endocrine, nutritional, and metabolic diseases and immunity disorders" (AHRQ CCS 48-58, event of interest); "other" (all other reasons as captured by AHRQ CCS); and no 


\section{TABLE 1 Cohort Baseline Characteristics}

\begin{tabular}{|c|c|c|c|c|c|}
\hline Characteristic & $\begin{array}{c}\text { All } \\
(\mathrm{N}=21,366)\end{array}$ & $\begin{array}{c}K<3.5 \mathrm{mEq} / \mathrm{L} \\
(\mathrm{n}=401)\end{array}$ & $\begin{array}{c}\text { K 3.5-5.5 mEq/L } \\
(n=20,295)\end{array}$ & $\begin{array}{c}\mathrm{K}>5.5 \mathrm{mEq} / \mathrm{L} \\
(\mathrm{n}=670)\end{array}$ & $P$ value \\
\hline Age at index & $68.6(10.4)$ & $65.6(10.2)$ & $68.6(10.4)$ & $70.7 \quad(10.2)$ & $<0.0001^{a}$ \\
\hline \multicolumn{6}{|l|}{ Sex } \\
\hline Males & $20,969 \quad(98.1)$ & 394 (98.3) & $19,917 \quad(98.1)$ & $658 \quad(98.2)$ & \multirow{2}{*}{$0.98^{\mathrm{b}}$} \\
\hline Female & $397 \quad(1.9)$ & $7 \quad(1.7)$ & $378 \quad(1.9)$ & $12 \quad(1.8)$ & \\
\hline \multicolumn{6}{|l|}{ Race } \\
\hline White & $14,726 \quad(68.9)$ & 189 (47.1) & $14,007 \quad(69.0)$ & $530(79.1)$ & \multirow{3}{*}{$<0.0001^{b}$} \\
\hline Black & $6,041 \quad(28.3)$ & $203(50.6)$ & $5,721 \quad(28.2)$ & 117 (17.5) & \\
\hline Other & $599 \quad(2.8)$ & $9 \quad(2.2)$ & $567 \quad(2.2)$ & $23 \quad(3.4)$ & \\
\hline Married & $12,329 \quad(57.7)$ & $216 \quad(54.1)$ & $11,689 \quad(57.6)$ & $424 \quad(63.3)$ & $0.009^{b}$ \\
\hline \multicolumn{6}{|l|}{ Region } \\
\hline Northeast & $3,153 \quad(14.7)$ & $36 \quad(8.9)$ & $3,004 \quad(14.8)$ & $113(16.9)$ & \multirow{5}{*}{$<0.0001^{\mathrm{b}}$} \\
\hline Midwest & $4,782 \quad(22.4)$ & $87 \quad(21.7)$ & $4,568 \quad(22.5)$ & $127 \quad(1.9)$ & \\
\hline South & $9,655 \quad(45.2)$ & $228 \quad(56.9)$ & $9,113 \quad(44.9)$ & $314(46.9)$ & \\
\hline West & $3,457 \quad(16.2)$ & $46 \quad(11.5)$ & $3,329 \quad(16.4)$ & $82 \quad(12.2)$ & \\
\hline Other & $305 \quad(1.4)$ & $267 \quad(1.3)$ & $4 \quad(1.0)$ & $34 \quad(5.1)$ & \\
\hline Income, \$ & $18,012(6,492-34,532)$ & $17,796(4,524-34,992)$ & $18,020(6,648-34,598)$ & $18,042(1,788-33,876)$ & $0.60^{c}$ \\
\hline \multicolumn{6}{|l|}{ Smoking status } \\
\hline Current & $6,930 \quad(32.5)$ & $121(30.2)$ & $6,613 \quad(32.6)$ & $196 \quad(29.3)$ & \multirow{3}{*}{$0.15^{b}$} \\
\hline Past & $7,529 \quad(35.3)$ & 134 (33.4) & $7,152 \quad(35.3)$ & $243(36.3)$ & \\
\hline Never & $6,893 \quad(32.3)$ & $146(36.4)$ & $6,516 \quad(32.1)$ & $231(34.5)$ & \\
\hline \multicolumn{6}{|l|}{ Comorbidities } \\
\hline Diabetes mellitus & $14,715 \quad(68.9)$ & $283(70.6)$ & $13,967 \quad(68.8)$ & $465 \quad(69.4)$ & $0.72^{\mathrm{b}}$ \\
\hline Congestive heart failure & $7,351 \quad(34.4)$ & $152(37.9)$ & $6,983 \quad(34.4)$ & $216 \quad(32.2)$ & $0.17^{\mathrm{b}}$ \\
\hline Hypertension & $20,668 \quad(96.7)$ & $390 \quad(97.3)$ & $19,639 \quad(96.8)$ & 639 (95.4) & $0.11^{\mathrm{b}}$ \\
\hline Peripheral vascular disease & $6,432 \quad(30.1)$ & $93 \quad(23.2)$ & $6,116 \quad(30.1)$ & $223(33.3)$ & $0.002^{\mathrm{b}}$ \\
\hline Cerebrovascular disease & $5,298 \quad(24.8)$ & $85(21.2)$ & $5,037 \quad(24.8)$ & $176(26.3)$ & $0.17^{b}$ \\
\hline Chronic lung disease & $6,207 \quad(29.1)$ & $104(25.9)$ & $5,915 \quad(29.2)$ & 188 (28.1) & $0.32^{\mathrm{b}}$ \\
\hline Peptic ulcer disease & $977 \quad(4.6)$ & $14 \quad(3.5)$ & $924 \quad(4.6)$ & $39 \quad(5.8)$ & $0.18^{\mathrm{b}}$ \\
\hline Ischemic heart disease & $10,477 \quad(49.0)$ & 189 (47.1) & $9,940 \quad(48.9)$ & $348 \quad(51.9)$ & $0.24^{\mathrm{b}}$ \\
\hline Paraplegia/hemiplegia & $492 \quad(2.3)$ & $7 \quad(1.8)$ & $474 \quad(2.3)$ & $11 \quad(1.6)$ & $0.38^{\mathrm{b}}$ \\
\hline Anemia & $9,990 \quad(46.8)$ & $164(40.9)$ & $9,476 \quad(46.7)$ & $350 \quad(52.2)$ & $0.001^{\mathrm{b}}$ \\
\hline Atrial fibrillation & $2,381 \quad(11.1)$ & $54(13.5)$ & $2,250 \quad(11.1)$ & 77 (11.5) & $0.31^{\mathrm{b}}$ \\
\hline Liver disease & $1,530 \quad(7.2)$ & $31 \quad(7.7)$ & $1,461 \quad(7.2)$ & $38 \quad(5.7)$ & $0.29^{b}$ \\
\hline Malignancies & $4,180 \quad(19.6)$ & $71 \quad(17.7)$ & $3,986 \quad(19.6)$ & $123(18.4)$ & $0.45^{b}$ \\
\hline
\end{tabular}

hospital visit (reference). We also assessed the association between categorized time-varying oK value with hospital visits due to "fluid and electrolyte disorders" (AHRQ CCS 55). For these analyses, the GEE model with a nominal multinomial distribution, a logit link, and an independent correlation matrix with the incrementally multivariable-adjusted models as listed in $\underline{\text { Supplementary Table } 1}$ were used. 


\section{TABLE 1 Cohort Baseline Characteristics (continued)}

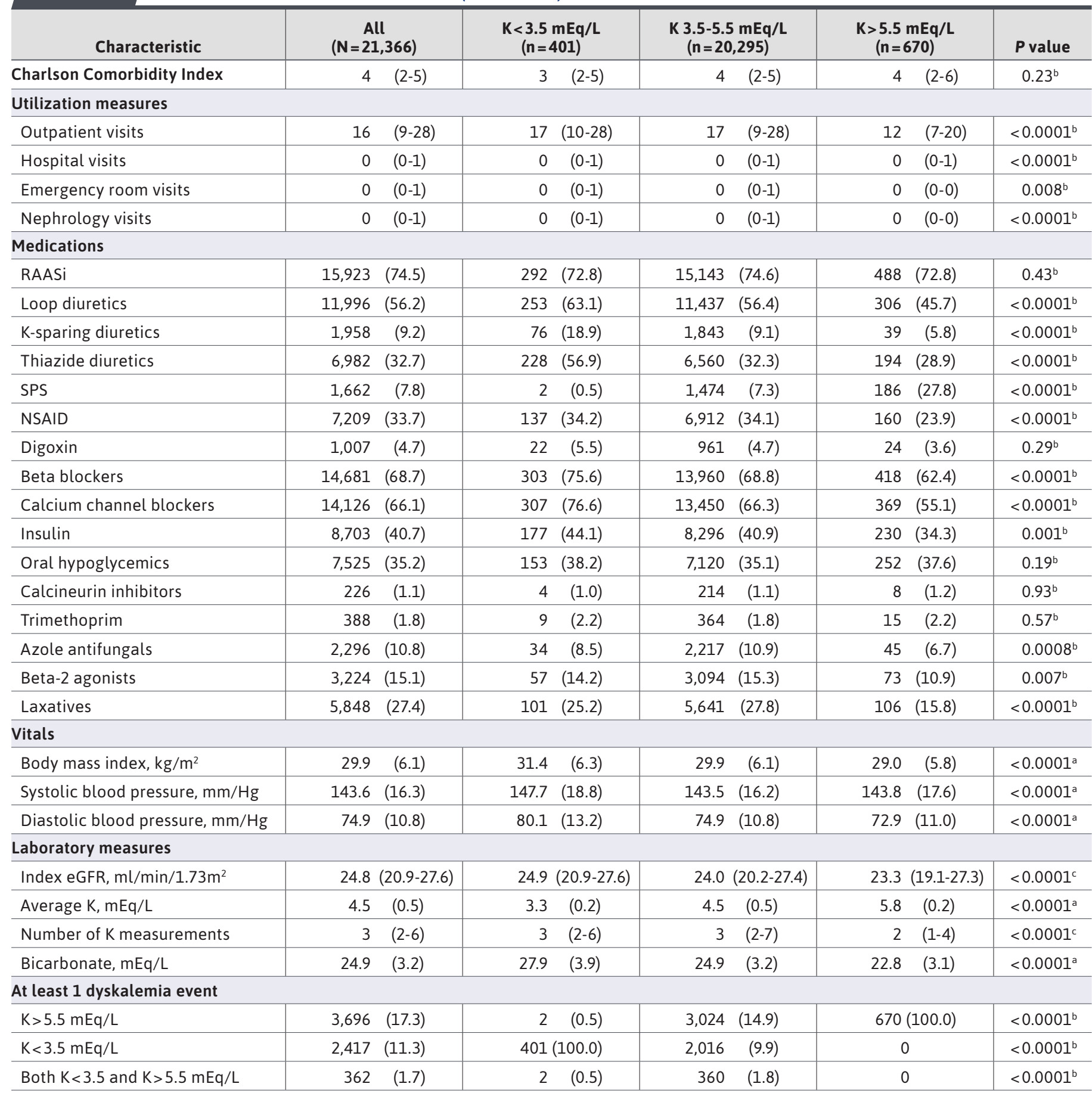




\begin{tabular}{|c|c|c|c|c|c|}
\hline Characteristic & $\begin{array}{c}\text { All } \\
(\mathrm{N}=21,366)\end{array}$ & $\begin{array}{c}\mathrm{K}<3.5 \mathrm{mEq} / \mathrm{L} \\
(\mathrm{n}=401)\end{array}$ & $\begin{array}{c}\text { K 3.5-5.5 mEq/L } \\
(n=20,295)\end{array}$ & $\begin{array}{c}K>5.5 \mathrm{mEq} / \mathrm{L} \\
(n=670)\end{array}$ & $P$ value \\
\hline \multicolumn{6}{|l|}{ Index eGFR year } \\
\hline 2005 & $3,459 \quad(16.2)$ & $41 \quad(10.2)$ & $3,289 \quad(16.2)$ & $129(19.3)$ & \multirow{10}{*}{$<0.0001^{b}$} \\
\hline 2006 & $3,322 \quad(15.6)$ & $53 \quad(13.2)$ & $3,143 \quad(15.5)$ & $126(18.8)$ & \\
\hline 2007 & $3,193 \quad(14.9)$ & $64 \quad(15.9)$ & $3,023 \quad(14.9)$ & $106(15.8)$ & \\
\hline 2008 & $2,758 \quad(12.9)$ & $59 \quad(14.7)$ & $2,607 \quad(12.8)$ & 92 (13.7) & \\
\hline 2009 & $2,474 \quad(11.6)$ & $56 \quad(13.9)$ & $2,349 \quad(11.6)$ & $69 \quad(10.3)$ & \\
\hline 2010 & $2,307 \quad(10.8)$ & $37 \quad(9.2)$ & $2,204 \quad(10.9)$ & $66 \quad(9.8)$ & \\
\hline 2011 & $1,853 \quad(8.7)$ & $44 \quad(10.9)$ & $1,765 \quad(8.7)$ & $44 \quad(6.6)$ & \\
\hline 2012 & $1,312 \quad(6.1)$ & $25 \quad(6.2)$ & $1,265 \quad(6.2)$ & $22 \quad(3.3)$ & \\
\hline 2013 & $657 \quad(3.1)$ & $22 \quad(5.5)$ & $619 \quad(3.0)$ & $16 \quad(2.4)$ & \\
\hline 2014 & $31 \quad(0.2)$ & 0 & $31 \quad(0.2)$ & 0 & \\
\hline \multicolumn{6}{|c|}{ 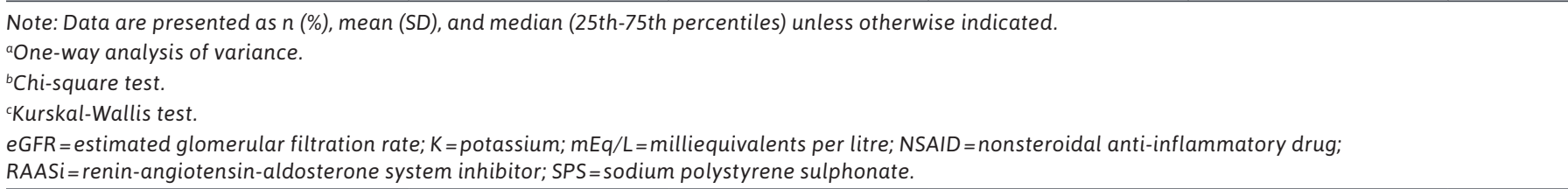 } \\
\hline
\end{tabular}

Missingness was observed for marital status (0.05\%), smoking status and region of residence (0.07\% each), systolic blood pressure (SBP) and diastolic blood pressure (DBP; 0.7\% each), body mass index (BMI; 0.8\%), bicarbonate (22.2\%), and time-varying eGFR (13.5\%). Data were assumed to be missing at random (MAR), since epidemiological studies are more likely to have such type of missing data compared with other missing data types, such as missing completely at random (MCAR) or missing not at random (MNAR). ${ }^{31-33}$ Further, the missing data pattern was found to be "arbitrary" as assessed by the proc MI procedure in SAS.

Baseline characteristics (marital status, smoking status, region of residence, $\mathrm{SBP}, \mathrm{DBP}, \mathrm{BMI}$, and bicarbonate) were imputed using single regression imputation, modeled on other complete covariates. Time-varying eGFR was imputed by estimating the eGFR values based on intra-individual eGFR slopes calculated from linear mixed-effects models using all available outpatient eGFR measured over the 1-year follow-up. The main analysis (models 1-6) was conducted using singly imputed data as previously described. We conducted several subgroup and sensitivity analyses to test the robustness of our results as follows:

1. Subgroup analyses were conducted after categorizing patients by age; race; region of residence; prevalent diabetes mellitus (DM) and congestive heart failure (CHF); baseline use of SPS, RAASi, and loop diuretics; and index eGFR using singly imputed data as previously described. Potential interactions between dyskalemia categories and selected subgroups were tested by including interactions terms.

2. The exposure-outcome association was assessed by categorizing the time-varying oK into granular categorizes as $\mathrm{K}<3.5 \mathrm{mEq} / \mathrm{L}, 3.5-<4.0 \mathrm{mEq} / \mathrm{L}, 4.0-<4.5 \mathrm{mEq} / \mathrm{L}$ (reference), $4.5-<5.0 \mathrm{mEq} / \mathrm{L}, 5.0-<5.5 \mathrm{mEq} / \mathrm{L}, 5.5-<6.0$ $\mathrm{mEq} / \mathrm{L}$, and $\geq 6.0 \mathrm{mEq} / \mathrm{L}$ using singly imputed data as previously described.

3. Analyses were repeated using multiple imputation (imputation $\mathrm{n}=25$ ) for baseline characteristics using the fully conditional specification method ${ }^{34,35}$ and complete cases ( $n=11,239$ for models 1-6) after excluding those with missing baseline and time-varying eGFR.

Compared with those included in the study cohort $(n=21,366)$, those excluded $(n=81,111)$ were older $(70.3$ vs 68.6 years); less likely to be males (91.9\% vs $98.1 \%$ ), African Americans (24.3\% vs $28.3 \%$ ), married (55.1\% vs $57.7 \%)$, and current smokers (19.8\% vs $32.5 \%)$; and have DM (59.5\% vs $68.9 \%)$.

A 2-sided $\mathrm{P}$ value of $<0.05$ was used as a threshold of statistical significance for all statistical analyses. All analyses were conducted in SAS Enterprise guide version 8.2 (SAS Institute) and STATA/MP version 15 (StataCorp). 


\section{FIGURE 1 Association of Time-Varying Outpatient Potassium with Hospital Visits}

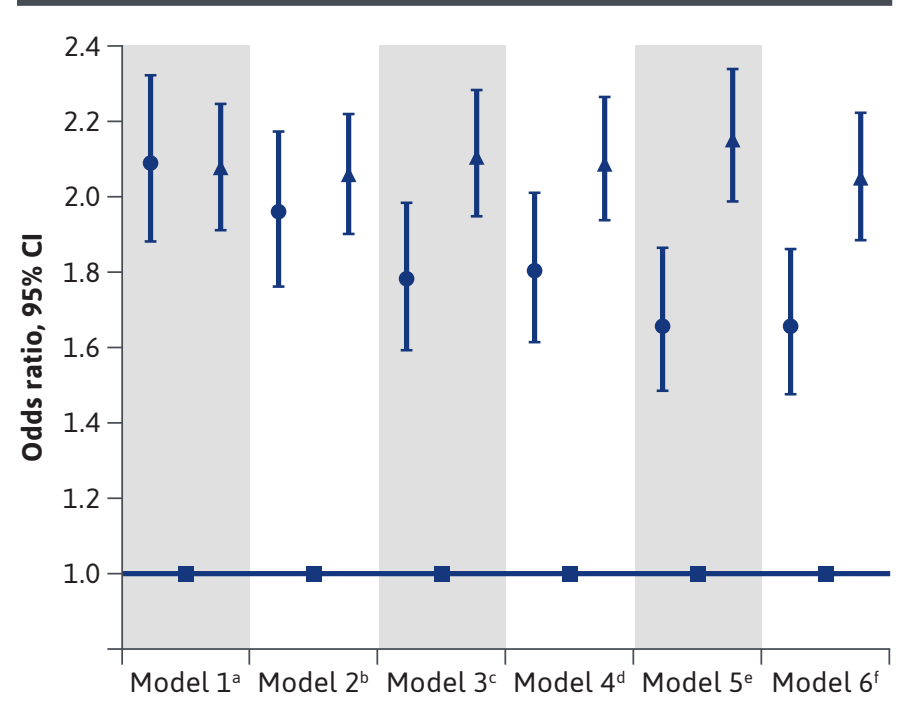

Referrent (K 3.5-5.5 mEq/L)

- Hypokalemia $(K<3.5 \mathrm{mEq} / \mathrm{L})$

Ayperkalemia $(K>5.5 \mathrm{mEq} / \mathrm{L})$

aModel 1: unadjusted.

bModel 2: adjusted for age, sex, race, marital status, region of residence, and income.

'Model 3: adjusted for model 2 and smoking status; systolic blood pressure; diastolic blood pressure; body mass index; comorbidities (diabetes mellitus, congestive heart failure, hypertension, ischemic heart disease, peripheral vascular disease, cerebrovascular disease, chronic lung disease, peptic ulcer, para-/hemiplegia, anemia, atrial fibrillation, liver disease, and malignancies); $\mathrm{CCl}$; and outpatient, hospital, emergency and nephrology visits measured at baseline.

'Model 4: adjusted for model 3 and baseline medications (RAASi, sodium polystyrene sulphonate, loop diuretics, K-sparing diuretics, thiazide diuretics, NSAIDs, digoxin, beta blockers, calcium channel blockers, insulin, oral hypoglycemics, calcineurin inhibitors, trimethoprim, azole antifungals, beta-2 agonists, and laxatives).

eModel 5: adjusted for model 4 and index eGFR, index year, $K$ levels and bicarbonate levels averaged over baseline, number of $K$ measurements, and at least 1 hyperkalemia event $(K>5.5 \mathrm{mEq} / \mathrm{L})$, hypokalemia event $(K<3.5 \mathrm{mEq} / \mathrm{L})$, and hypo-/hyperkalemia event measured over baseline.

'Model 6: adjusted for model 5 and time-varying medications listed above and time-varying outpatient eGFR.

$\mathrm{CCl}=$ Charlson Comorbidity Index; eGFR=estimated glomerular filtration rate; $\mathrm{K}=$ serum potassium; $\mathrm{mEq} / \mathrm{L}=$ milliequivalents per litre; $N S A I D=$ nonsteroidal anti-inflammatory drug; RAASi = renin-angiotensin-aldosterone system inhibitor.

This study was approved by the institutional review boards of the Memphis and Long Beach VA Medical Centers, with exemption from informed consent.

\section{Results}

\section{BASELINE CHARACTERISTICS}

The mean (SD) age of the sample was 68.6 (10.4) years; $98.1 \%$ were males; 28.3\% were African Americans; and 68.9\% had DM (Table 1). The most commonly used medications were RAASi $(74.5 \%)$, beta blockers (68.7\%), and calcium channel blockers (66.1\%). Approximately $8 \%$ of the patients used SPS. The median (25th-75th percentiles) index eGFR was 24.8 (20.9-27.6) $\mathrm{ml} / \mathrm{min} / 1.73 \mathrm{~m}^{2}$. Patients had a median (25th-75th percentiles) of 3 (2-6) K measurements, with a mean (SD) K of $4.5(0.5) \mathrm{mEq} / \mathrm{L}$ in the baseline. At least 1 hyperkalemia and hypokalemia event in the baseline was experienced by $17.3 \%$ and $11.3 \%$ patients, respectively. Approximately $3 \%$ and $1.9 \%$ of the sample had average baseline $\mathrm{K}$ levels $>5.5$ $\mathrm{mEq} / \mathrm{L}$ and $<3.5 \mathrm{mEq} / \mathrm{L}$, respectively. Those with average baseline $\mathrm{K}$ levels $>5.5 \mathrm{mEq}$ /L were more likely (all $\mathrm{P}$ values $<0.05$ ) to be older, White, married, have prevalent peripheral vascular disease and anemia, SPS users, and have lower eGFR, bicarbonate, SBP, DBP, and BMI levels.

\section{EXPOSURE POTASSIUM CHARACTERISTICS}

During the 1-year follow-up period, 125,266 oK values were observed, with a median (25th-75th percentiles) of 5 (2-8) measurements per patient, of which $6.8 \%$ and $3.7 \%$ were categorized as hyper- and hypokalemia, respectively.

\section{ASSOCIATION OF DYSKALEMIAS WITH HOSPITAL VISITS}

Over the 1-year follow-up period, 8,635 (40\%) patients experienced a hospital visit, with a total (median [25th-75th percentiles]) of 18,374 (2 [1-3]) hospital visits. Across the 125,266 oK measurements in the 1-year follow-up period, 8,542 (6.8\%; proportion $=0.068,95 \% \mathrm{CI}=0.067-0.069)$ hospital visits were observed within 2 calendar days of the measured $\mathrm{oK}$. In the unadjusted analysis, hyperkalemia (odds ratio $[\mathrm{OR}]=2.08 ; 95 \% \mathrm{CI}=1.92-2.24$ ) and hypokalemia $(\mathrm{OR}=2.09 ; 95 \% \mathrm{CI}=1.89-2.32)$ were associated with significantly higher odds of a hospital visit (Figure 1, Model 1; Supplementary Table 2, Model 1, available in online article).

In the multivariable-adjusted model, although the point estimates were slightly attenuated, hyperkalemia $(\mathrm{OR}=2.04$; $95 \% \mathrm{CI}=1.88-2.21)$ and hypokalemia $(\mathrm{OR}=1.66 ; 95 \% \mathrm{CI}=1.48-$ 1.86) were associated with significantly higher odds of a hospital visit (Figure 1, Model 6; Supplementary Table 2. Model 6). In the multivariable-adjusted model, the association of granular $\mathrm{K}$ value categories showed a U-shaped association with hospital visits, with significantly higher odds of a hospital visit (vs K 4.0 to $<4.5 \mathrm{mEq} / \mathrm{L}$ ) associated with $\mathrm{K}<3.5 \mathrm{mEq} / \mathrm{L}(\mathrm{OR}=1.71 ; 95 \% \mathrm{CI}=1.51-1.92)$ and $\mathrm{K} \geq 6.0$ $\mathrm{mEq} / \mathrm{L}(\mathrm{OR}=3.56 ; 95 \% \mathrm{CI}=3.15-4.02$; Model 6 ; Supplementary 


\section{FIGURE 2 Association of Time-Varying Outpatient Potassium with Hospital Visits by Subgroups}

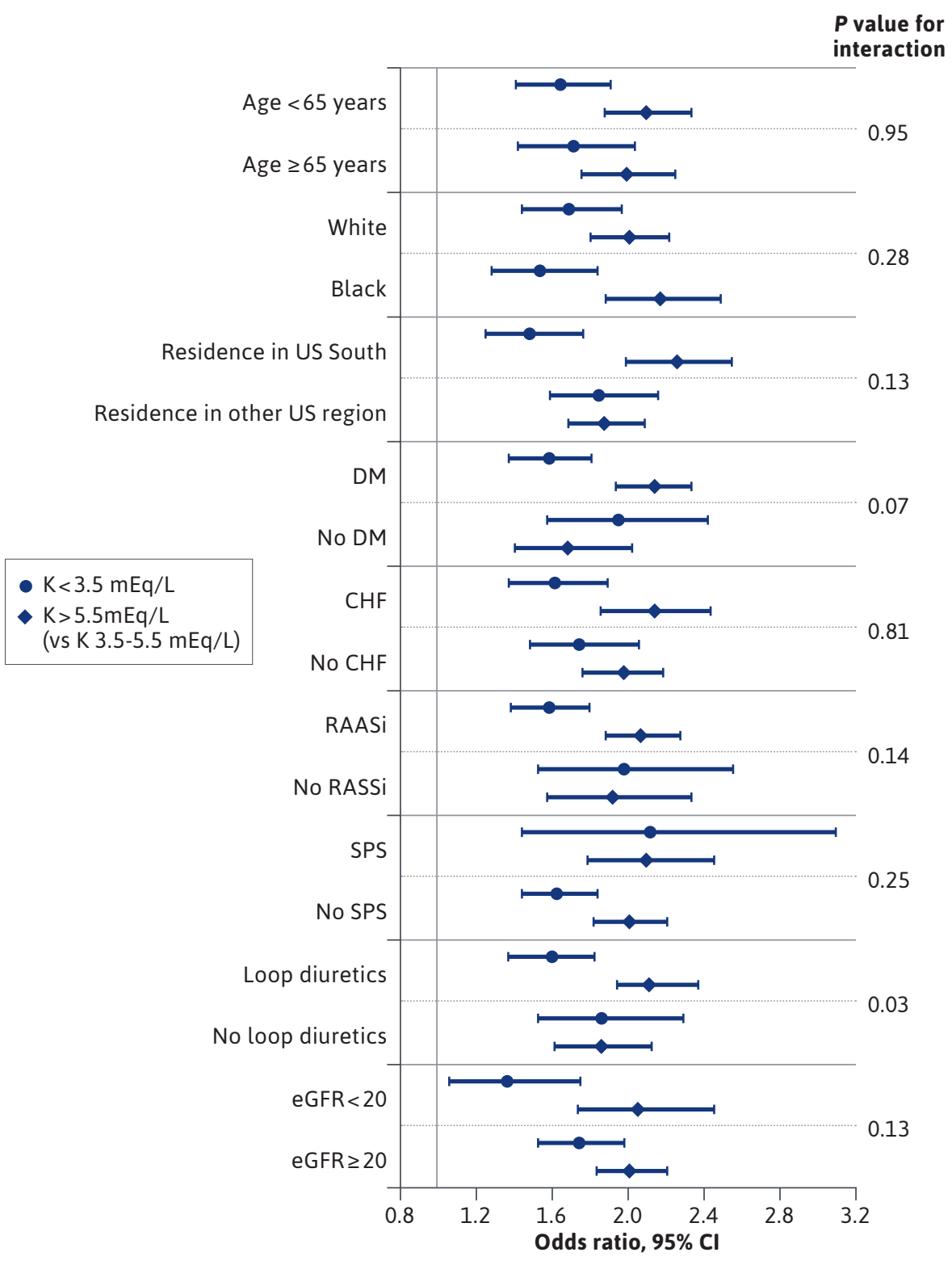

Note: Model adjusted for age; sex; race; marital status; region of residence and income; smoking status; systolic blood pressure and diastolic blood pressure; body mass index; comorbidities (diabetes mellitus, congestive heart failure, hypertension, ischemic heart disease, peripheral vascular disease, cerebrovascular disease, chronic lung disease, peptic ulcer, para/hemiplegia, anemia, atrial fibrillation, liver disease, and malignancies); CCl; outpatient, hospital, emergency and nephrology visits measured at baseline; baseline medications (RAASi, sodium polystyrene sulphonate, loop diuretics, K-sparing diuretics, thiazide diuretics, NSAIDs, digoxin, beta blockers, calcium channel blockers, insulin, oral hypoglycemics, calcineurin inhibitors, trimethoprim, azole antifungals, beta-2 agonists, and laxatives); index eGFR; index year; $K$ levels and bicarbonate levels averaged over baseline; number of $K$ measurements; and at least 1 hyperkalemia event $(K>5.5 \mathrm{mEq} / \mathrm{L})$, hypokalemia event $(K<3.5 \mathrm{mEq} / \mathrm{L})$, and hypo/hyperkalemia event measured over baseline, time-varying medications listed above, and time-varying outpatient eGFR.

$\mathrm{CCl}=$ Charlson Comorbidity Index; $\mathrm{CHF}=$ congestive heart failure; $\mathrm{DM}=$ diabetes mellitus; $e G F R=$ estimated glomerular filtration rate; $K=$ serum potassium; NSAID = nonsteroidal anti-inflammatory drug; RAASi = renin-angiotensin-aldosterone system inhibitor; SPS=sodium polystyrene sulphonate.
Table 3, available in online article). The association of dyskalemias with hospital visits was similar using multiple imputation and complete case analyses (Supplementary Table 4A and $\underline{4 B}$, respectively, available in online article).

Among the 8,542 hospital visits, $36 \%$ were due to "diseases of the circulatory system" (of which 5.6\% [172 of 3,078] were due to "arrhythmias"); $15.2 \%$ were due to "diseases of the genitourinary system"; and $12.2 \%$ were due to "endocrine, nutritional, and metabolic diseases and immunity disorders" (of which 34.3\% [356 of 1,039] were due to "fluid and electrolyte disorders"; Supplementary Table 5, available in online article). In the multivariable-adjusted model, hyperkalemia $(\mathrm{OR}=1.39 ; 95 \% \mathrm{CI}=1.20$ 1.63) and hypokalemia $(\mathrm{OR}=1.52 ; 95 \%$ $\mathrm{CI}=1.28-1.79)$ were associated with higher odds of a hospital visit due to "diseases of the circulatory system" but showed a nonsignificant higher odds of hospital visits due to "arrhythmias" (Supplementary Table 6A and $\underline{6 B}$, Model 6 , respectively, available in online article).

Similarly, in the multivariableadjusted model, hyperkalemia $(\mathrm{OR}=4.47 ; 95 \% \mathrm{CI}=3.82-5.23)$ and hypokalemia $(\mathrm{OR}=2.43 ; 95 \% \mathrm{CI}=1.83-3.21)$ were associated with higher odds of a hospital visit due to "endocrine, nutritional, and metabolic diseases and immunity disorders" (Supplementary Table 7A, Model 6, available in online article). Specifically, hyperkalemia $(\mathrm{OR}=11.86 ; 95 \% \mathrm{CI}=9.30-15.11)$ and hypokalemia $(\mathrm{OR}=4.44 ; 95 \% \mathrm{CI}=2.77$ 7.14) were associated with higher odds of hospital visit due to "fluid and electrolyte disorders" (Supplementary Table 7B, Model 6). In the subgroup analysis, significant differences were found by the loop diuretic users subgroup, with those using loop diuretics having lower odds of a hospital visit following a hypokalemia event 


\section{FIGURE 3 Association of Time-Varying Outpatient Potassium with Emergency Room Visits}

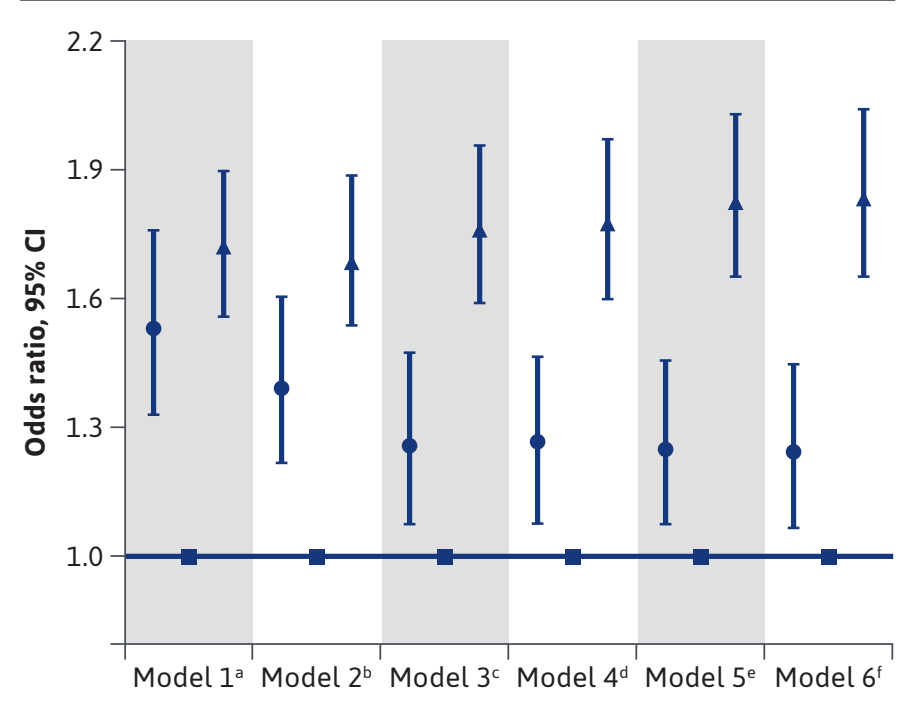

Referrent (K 3.5-5.5 mEq/L)

- Hypokalemia $(K<3.5 \mathrm{mEq} / \mathrm{L})$

A Hyperkalemia $(\mathrm{K}>5.5 \mathrm{mEq} / \mathrm{L})$

aModel 1: unadjusted.

bModel 2: adjusted for age, sex, race, marital status, region of residence, and income.

'Model 3: adjusted for model 2 and smoking status; systolic blood pressure; diastolic blood pressure; body mass index; comorbidities (diabetes mellitus, congestive heart failure, hypertension, ischemic heart disease, peripheral vascular disease, cerebrovascular disease, chronic lung disease, peptic ulcer, para-/hemiplegia, anemia, atrial fibrillation, liver disease, and malignancies); $\mathrm{CCl}$; and outpatient, hospital, emergency and nephrology visits measured at baseline.

'Model 4: adjusted for model 3 and baseline medications (RAASi, sodium polystyrene sulphonate, loop diuretics, K-sparing diuretics, thiazide diuretics, NSAIDs, digoxin, beta blockers, calcium channel blockers, insulin, oral hypoglycemics, calcineurin inhibitors, trimethoprim, azole antifungals, beta-2 agonists, and laxatives).

eModel 5: adjusted for model 4 and index eGFR, index year, $K$ levels and bicarbonate levels averaged over baseline, number of $K$ measurements, and at least 1 hyperkalemiaevent $(K>5.5 \mathrm{mEq} / \mathrm{L})$, hypokalemiaevent $(\mathrm{K}<14113.5 \mathrm{mEq} / \mathrm{L})$, and hypo-/hyperkalemia event measured over baseline.

'Model 6: adjusted for model 5 and time-varying medications listed above and time-varying outpatient eGFR.

$\mathrm{CCl}=$ Charlson Comorbidity Index; eGFR = estimated glomerular filtration rate $\mathrm{K}=$ serum potassium; $\mathrm{mEq} / \mathrm{L}=$ milliequivalents per litre; $N S A I D=$ nonsteroidal anti-inflammatory drug; $R A A S i=$ renin-angiotensin-aldosterone system inhibitor. (vs hypokalemia in nonusers; Figure 2 and Supplementary Table 8, available in online article).

\section{ASSOCIATION OF DYSKALEMIAS WITH ER VISITS}

Over the 1-year follow-up period, 7,135 (33.4\%) patients experienced an ER visit, with a total (median [25th-75th percentiles]) of 14,368 (1 [1-2]) ER visits. Across the 125,266 oK measurements in the 1-year follow-up period, 5,114 (4.1\%; proportion $=0.041,95 \% \mathrm{CI}=0.039-0.042)$ ER visits were observed within 2 calendar days of the measured oK. In the unadjusted analysis, hyperkalemia $(\mathrm{OR}=1.72 ; 95 \%$ $\mathrm{CI}=1.56-1.89)$ and hypokalemia $(\mathrm{OR}=1.52 ; 95 \% \mathrm{CI}=1.33-1.75)$ were associated with significantly higher odds of an ER visit (Figure 3, Model 1 and Supplementary Table 9, Model 1, available in online article).

Similarly, in the multivariable-adjusted model, hyperkalemia $(\mathrm{OR}=1.83 ; 95 \% \mathrm{CI}=1.65-2.03)$ and hypokalemia $(\mathrm{OR}=1.24 ; 95 \% \mathrm{CI}=1.07-1.44)$ were associated with significantly higher odds of an ER visit (Figure 3, Model 6 and Supplementary Table 9, Model 6). In the multivariable-adjusted model, the association of granular $\mathrm{K}$ value categories showed a U-shaped association with ER visits, with significantly higher odds of an ER visit (vs K 4.0 to $<4.5 \mathrm{mEq} / \mathrm{L})$ associated with $\mathrm{K}<3.5 \mathrm{mEq} / \mathrm{L}(\mathrm{OR}=1.20 ; 95 \%$ $\mathrm{CI}=1.03-1.40)$ and $\mathrm{K} \geq 6.0 \mathrm{mEq} / \mathrm{L}(\mathrm{OR}=3.04 ; 95 \% \mathrm{CI}=2.61-$ 3.54; Supplementary Table 10, Model 6, available in online article). The association of dyskalemias with ER visits was similar, using multiple imputation and complete case analyses (Supplementary Table 11A and 11B, respectively, available in online article).

In the subgroup analysis, significant differences were observed by region of residence (South vs other US regions; Figure 4 and Supplementary Table 12, available in online article). The odds of an ER visit following a hypokalemia event was significantly higher among those residing in the US South.

\section{Discussion}

In a nationally representative cohort of US veterans with advanced CKD who transitioned to dialysis, dyskalemias were associated with higher risk of short-term hospital and ER visits in the predialysis period. Overall, the risk for such short-term visits was higher following a hyperkalemia event compared with a hypokalemia event. In addition, the risk for a hospital visit was higher compared with emergency visits, with higher risk associated with hyperkalemia than hypokalemia. When K levels were categorized into granular categories, the risk for short-term visits was the highest at the extreme K levels. 


\section{FIGURE 4 Association of Time-Varying Outpatient Potassium with Emergency Room Visits by Subgroups}

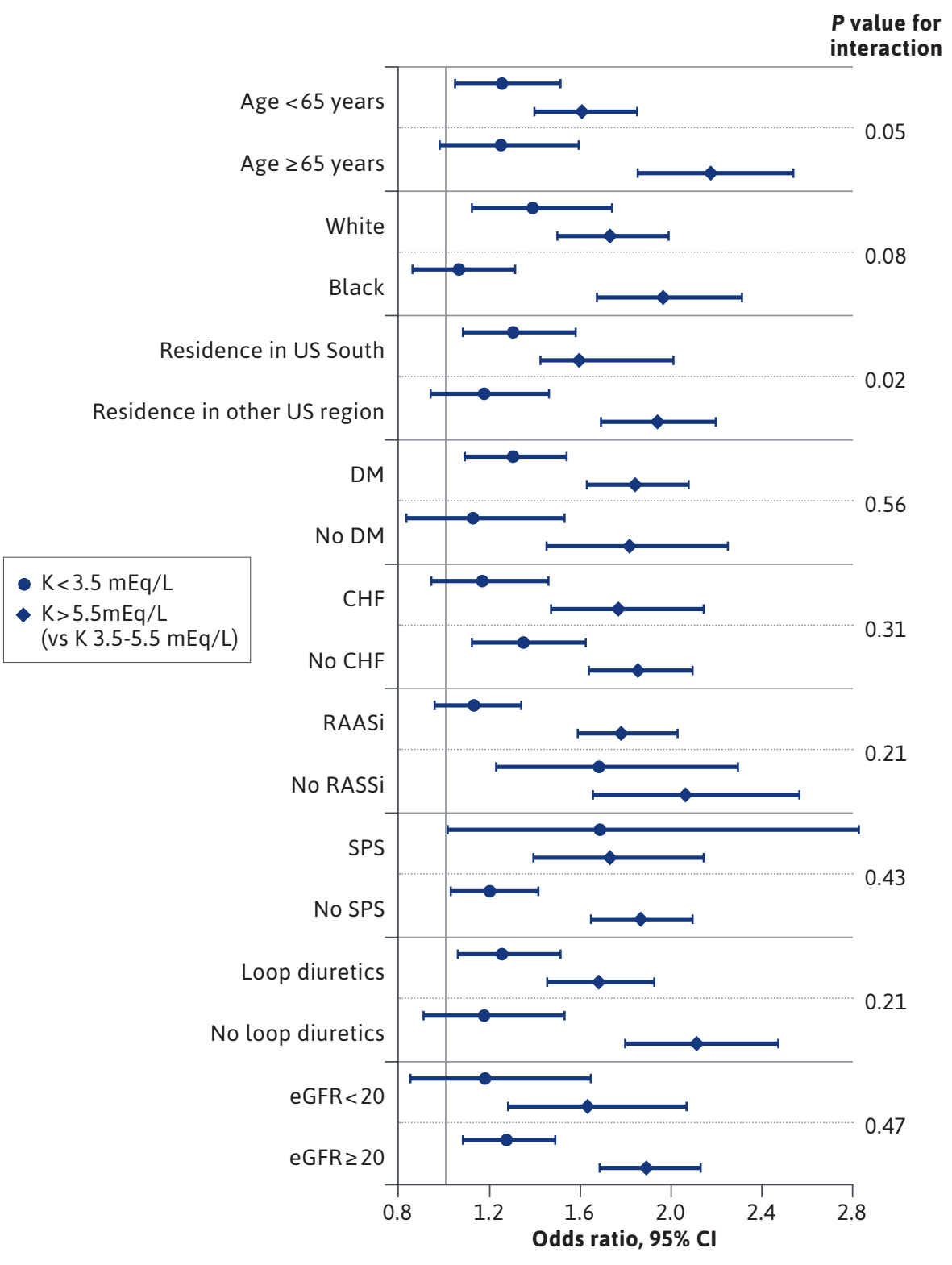

Note: Model adjusted for age; sex; race; marital status; region of residence and income; smoking status; systolic blood pressure and diastolic blood pressure; body mass index; comorbidities (diabetes mellitus, congestive heart failure, hypertension, ischemic heart disease, peripheral vascular disease, cerebrovascular disease, chronic lung disease, peptic ulcer, para/hemiplegia, anemia, atrial fibrillation, liver disease, and malignancies); CCl; outpatient, hospital, emergency and nephrology visits measured at baseline; baseline medications (RAASi, sodium polystyrene sulphonate, loop diuretics, K-sparing diuretics, thiazide diuretics, NSAIDs, digoxin, beta blockers, calcium channel blockers, insulin, oral hypoglycemics, calcineurin inhibitors, trimethoprim, azole antifungals, beta-2 agonists, and laxatives); index eGFR; index year; $K$ levels and bicarbonate levels averaged over baseline; number of $K$ measurements; and at least 1 hyperkalemia event $(K>5.5 \mathrm{mEq} / \mathrm{L})$, hypokalemia event $(K<3.5 \mathrm{mEq} / \mathrm{L})$, and hypo-/hyperkalemia event measured over baseline, time-varying medications listed above, and time-varying outpatient eGFR.

$\mathrm{CCl}=$ Charlson Comorbidity Index; $\mathrm{CHF}=$ congestive heart failure; $\mathrm{DM}=$ diabetes mellitus; $e G F R=$ estimated glomerular filtration rate; $\mathrm{K}=$ serum potassium; NSAID = nonsteroidal anti-inflammatory drug;

RAASi = renin-angiotensin-aldosterone system inhibitors; SPS =sodium polystyrene sulphonate.
Dyskalemias were associated with higher risk of hospital visits due to "diseases of the circulatory system" and "endocrine, nutritional, and metabolic diseases and immunity disorders," especially the latter. Further, for hospital visits due to "arrhythmias," there was a nonsignificant higher risk associated with dyskalemias. Also, hypo- and hyperkalemia were associated with a 4.4- and 11.9fold higher risk for hospital visits due to "fluid and electrolyte disorders" (which includes hypo- and hyperkalemia), respectively. However, caution should be exercised when interpreting the association of dyskalemias with hospital visit subtypes due to the low event rates and wide CIs.

Our results align with existing literature that has assessed the association of dyskalemias (baseline and time-varying exposure) with health care utilization (as a distant/ immediate outcome)..$^{5,11,13-16}$ Among those with eGFR $<30 \mathrm{ml} / \mathrm{min} / 1.73 \mathrm{~m}^{2},{ }^{5}$ time-varying $\mathrm{K} 5.5-5.9 \mathrm{mEq} / \mathrm{L}, \mathrm{K} \geq 6.0$ $\mathrm{mEq} / \mathrm{L}$, and $\mathrm{K}<3.5 \mathrm{mEq} / \mathrm{L}$ were associated with a 1.3-, 3.7-, and 1.9fold (vs K 4.5-4.9 mEq/L) higher risk of a hospital visit, respectively. In a hemodialysis cohort, ${ }^{13}$ Brunelli et al observed a higher risk of hospital and ER visits with increasing levels of $\mathrm{K}$ (vs $\mathrm{K} 4.0$ to $<4.5 \mathrm{mEq} / \mathrm{L}$ ), with a higher risk associated with a hospital visit compared with an ER visit. Further, similar to Brunelli et al, we observed that hospital visits due to "diseases of the circulatory system" visits were the most common (36\% in our study vs $28.7 \%$ ), and the number of hospital visits due to "endocrine, nutritional, and metabolic diseases and immunity disorders" were also similar ( $12 \%$ in our study vs $\sim 9 \%)$. Finally, similar to Brunelli et al, we observed a higher risk associated with higher $\mathrm{K}$ levels for hospital visits due to "diseases of the circulatory system" and "endocrine, nutritional, and metabolic diseases 
and immunity disorders," especially for the latter.

The risk of dyskalemias increases as kidney function worsens, and further dyskalemias are associated with higher risk of cardiovascular events and mortality. 1,3-6,10,12,36 Thus, patients are at a higher risk of hospital/ER visits due to dyskalemia-induced events such as arrhythmias or are simply referred to an ER or hospitalized after detection of a dyskalemia event for fear of a potential cardiovascular event.

Our study has several clinical implications. Studies in CKD/ ESRD populations have shown that those experiencing hyperkalemia incur higher hospital/ER visit costs compared with those who do not experience hyperkalemia. ${ }^{11,13-16}$ Studies quantifying health care costs associated with hypokalemia are currently lacking, but it can be assumed that hypokalemia represents a substantial economic burden. We can hypothesize that correcting and especially preventing dyskalemias could improve clinical outcomes and reduce hospital/ER visits and associated costs. Among patients experiencing hyperkalemia A, a study by Toto et al found that risk of hospital visits was lower (vs no medication use) with the use of SPS and patiromer (especially the latter) and the risk of ER visits was lower with patiromer use. ${ }^{37}$ This finding further emphasizes that correcting/ preventing hyperkalemia (and potentially even hypokalemia) can indeed reduce health care burden and associated costs.

While the management of hypokalemia is straightforward (K supplementation, diuretic dose reduction, changing antihypertensives from diuretics to RAASi or K-sparing diuretics), the management of hyperkalemia is more complex (including acute interventions such as administration of insulin, glucose, beta-agonists, and intravenous sodium bicarbonate, or chronic interventions such as the use of $\mathrm{K}$ binding agents or discontinuation of RAASi)., ${ }^{1,2}$ The advent of newer K-binding agents, such as patiromer calcium and sodium zirconium cyclosilicate for chronic hyperkalemia, might help reduce the incidence of hyperkalemia with progressive decline in kidney function and could allow uninterrupted use of cardioand renoprotective agents such as RAASi, ultimately leading to improved clinical outcomes. ${ }^{38}$ The prevalence of hypokalemia is comparatively lower than hyperkalemia and the treatment is less complex ${ }^{3,8}$; however, the health care burden and costs need to be considered when managing patients experiencing hypokalemia.

\section{LIMITATIONS}

Our study results need to be interpreted in light of several limitations. First, we used observational data, hence, we cannot infer causality but only associations.

Second, the cohort consisted of predominantly male US veterans (98\%), so the results may not be generalizable to women or a broader general population.

Third, all patients survive until dialysis initiation due to the cohort construction, and we studied the exposure-outcome relationship in the predialysis period. However, our results align with previous literature, thus, validating the generalizability of our results.

Fourth, we assumed the missing data to be MAR, since this missing type seems more likely to hold true in epidemiological studies compared with other types, such as MCAR or MNAR. ${ }^{31-33}$ The MAR assumption allowed for the use of a single regression imputation (and even multiple imputation) method for imputing missing data based on observed complete covariates. While the MAR assumption may not necessarily hold true (owing to unmeasured confounders), the similarity of our results across the single imputation, multiple imputation, and complete case analyses further validates the robustness of our study results and missing data assumptions.

Finally, because of the nature of the study, we cannot eliminate the possibility of unmeasured confounders such as a lack of data regarding dietary $\mathrm{K}$ intake and other nutritional markers.

\section{Conclusions}

In patients with advanced CKD who are transitioning to dialysis, dyskalemias are associated with a higher risk of hospital and ER visits. Close monitoring of $\mathrm{K}$ levels and the advent of newer K-binding agents (in the context of hyperkalemia) might help in reducing health care utilization and eventual economic burden.

\section{DISCLOSURES}

This study was supported by grant 5U01DK102163 from the National Institute of Health (NIH) to Kamyar Kalantar-Zadeh and Csaba P. Kovesdy and by resources from the US Department of Veterans Affairs. The data reported here have been supplied in part by the United States Renal Data System (USRDS). Support for VA/ CMS data were provided by the Department of Veterans Affairs, Veterans Health Administration, Office of Research and Development, Health Services Research and Development, VA Information Resource Center (project numbers SDR 02-237 and 98-004). Opinions expressed in this article are those of the authors and do not necessarily represent the opinion of the Department of Veterans Affairs or the funding institution.

Kovesdy has received honoraria from Akebia, Ardelyx, Astra Zeneca, Bayer, Boehringer-Ingelheim, Cara Therapeutics, Reata, and Tricida unrelated to this study. Kalantar-Zadeh has received honoraria and/or support from Abbott, 
Abbvie, ACI Clinical (Cara Therapeutics), Akebia, Alexion, Amgen, American Society of Nephrology, Astra-Zeneca, Aveo, BBraun, Chugai, Cytokinetics, Daiichi, DaVita, Fresenius, Genentech, Haymarket Media, Hofstra Medical School, International Federation of Kidney Foundations, International Society of Hemodialysis, International Society of Renal Nutrition \& Metabolism, Japanese Society of Dialysis Therapy, Hospira, Kabi, Keryx, Kissei, Novartis, OPKO, National Institutes of Health, National Kidney Foundations, Pfizer, Regulus, Relypsa, Resverlogix, Dr Schaer, Sandoz, Sanofi, Shire, Veterans Affairs, Vifor, UpToDate, and ZS-Pharma, unrelated to this study.

Gatwood has received research support from AstraZeneca, Merck \& Co., and GlaxoSmithKline unrelated to this study. Obi has received research support from Relypsa/Vifor Pharma Inc. The remaining authors declare that they have no relevant financial interests.

\section{REFERENCES}

1. Kovesdy CP, Appel LJ, Grams ME, et al. Potassium homeostasis in health and disease: a scientific workshop cosponsored by the National Kidney Foundation and the American Society of Hypertension. Am J Kidney Dis. 2017;70(6):844-58.

2. Palmer BF, Clegg DJ. Physiology and pathophysiology of potassium homeostasis: core curriculum 2019. Am J Kidney Dis. 2019;74(5):682-95.

3. Gilligan S, Raphael KL. Hyperkalemia and hypokalemia in CKD: prevalence, risk factors, and clinical outcomes. Adv Chronic Kidney Dis. 2017;24(5):315-18.

4. Korgaonkar S, Tilea A, Gillespie BW, et al. Serum potassium and outcomes in CKD: insights from the RRI-CKD cohort study. Clin J Am Soc Nephrol. 2010;5(5):762-69.

5. Luo J, Brunelli SM, Jensen DE, Yang A. Association between serum potassium and outcomes in patients with reduced kidney function. Clin J Am Soc Nephrol. 2016;11(1):90-100.

6. Nakhoul GN, Huang H, Arrigain S, et al. Serum potassium, end-stage renal disease and mortality in chronic kidney disease. Am J Nephrol. 2015;41(6):456-63.
7. Moranne O, Froissart M, Rossert J, et al. Timing of onset of CKD-related metabolic complications. J Am Soc Nephrol. 2009;20(1):164-71.

8. Dashputre AA, Sumida K, Potukuchi PK, et al. Potassium trajectories prior to dialysis and mortality following dialysis initiation in patients with advanced CKD. Nephron. 2021;145(3):265-74.

9. McCullough PA, Beaver TM, Bennett-Guerrero E, et al. Acute and chronic cardiovascular effects of hyperkalemia: new insights into prevention and clinical management. Rev Cardiovasc Med. 2014;15(1):11-23.

10. Collins AJ, Pitt B, Reaven N, et al. Association of serum potassium with all-cause mortality in patients with and without heart failure, chronic kidney disease, and/or diabetes. Am J Nephrol. 2017;46(3):213-21.

11. Fitch K, Woolley JM, Engel T, Blumen H. The clinical and economic burden of hyperkalemia on medicare and commercial payers. Am Health Drug Benefits. 2017;10(4):202-10.

12. Kovesdy CP, Matsushita K, Sang Y, et al. Serum potassium and adverse outcomes across the range of kidney function: a CKD Prognosis Consortium meta-analysis. Eur Heart J. 2018;39(17):1535-42.

13. Brunelli SM, Du Mond C, Oestreicher N, Rakov V, Spiegel DM. Serum potassium and short-term clinical outcomes among hemodialysis patients: impact of the long interdialytic interval. Am J Kidney Dis. 2017;70(1):21-29.

14. Kim K, Thomsen RW, Nicolaisen SK, Hasvold LP, Palaka E, Sorensen HT. Healthcare resource utilisation and cost associated with elevated potassium levels: a Danish population-based cohort study. BMJ Open. 2019;9(4):e026465.

15. Polson M, Lord TC, Kangethe A, et al. Clinical and economic impact of hyperkalemia in patients with chronic kidney disease and heart failure. J Manag Care Spec Pharm. 2017;23(4-a Suppl):S2-S9. doi: 10.18553/jmcp.2017.23.4-a.s2a
16. Sharma A, Alvarez PJ, Woods SD, Fogli J, Dai D. Healthcare resource utilization and costs associated with hyperkalemia in a large managed care population. J Pharm Health Serv Res. 2021;12(1):35-41.

17. Saran R, Robinson B, Abbott KC, et al. US Renal Data System 2018 annual data report: epidemiology of kidney disease in the United States. Am J Kidney Dis. 2019;73(3S1):A7-A8.

18. Gaipov A, Molnar MZ, Potukuchi PK, et al. Acute kidney injury following coronary revascularization procedures in patients with advanced CKD. Nephrol Dial Transplant. 2019;34(11):1894-901.

19. Sumida K, Diskin CD, Molnar MZ, et al. Pre-end-stage renal disease hemoglobin variability predicts post-end-stage renal disease mortality in patients transitioning to dialysis. Am J Nephrol. 2017;46(5):397-407.

20. Sumida K, Molnar MZ, Potukuchi PK, et al. Pre-end-stage renal disease visitto-visit systolic blood pressure variability and post-end-stage renal disease mortality in incident dialysis patients.

J Hypertens. 2017;35(9):1816-24.

21. Sumida K, Molnar MZ, Potukuchi PK, et al. Blood pressure before initiation of maintenance dialysis and subsequent mortality. Am J Kidney Dis. 2017;70(2):207-17.

22. Dashputre AA, Potukuchi PK, Sumida K, et al. Pre-dialysis potassium variability and post-dialysis mortality in patients with advanced CKD. Kidney Int Rep. 2021;6(2):366-80.

23. Sumida K, Dashputre AA, Potukuchi PK, et al. Laxative use and risk of dyskalemia in patients with advanced ckd transitioning to dialysis. J Am Soc Nephrol. 2021;32(4):950-59.

24. McGinnis KA, Brandt CA, Skanderson $\mathrm{M}$, et al. Validating smoking data from the Veteran's Affairs Health Factors dataset, an electronic data source. Nicotine Tob Res. 2011;13(12):1233-39. 
25. Soohoo M, Moradi H, Obi Y, et al. Statin therapy before transition to end-stage renal disease with posttransition outcomes. J Am Heart Assoc. 2019;8(6):e011869.

26. Deyo RA, Cherkin DC, Ciol MA. Adapting a clinical comorbidity index for use with ICD-9-CM administrative databases. J Clin Epidemiol. 1992;45(6):613-19.

27. Kovesdy CP, Norris KC, Boulware LE, et al. Association of race with mortality and cardiovascular events in a large cohort of US veterans. Circulation. 2015;132(16):1538-48.

28. Kovesdy CP, Alrifai A, Gosmanova EO, et al. Age and outcomes associated with $\mathrm{BP}$ in patients with incident CKD. Clin J Am Soc Nephrol. 2016;11(5):821-31.

29. Levey AS, Stevens LA, Schmid CH, et al. A new equation to estimate glomerular filtration rate. Ann Intern Med. 2009;150(9):604-12.
30. Healthcare Cost and Utilization Project (HCUP). Clinical Classifications Software (CCS) for ICD-9-CM. March 2017. Agency for Healthcare Research and Quality. Accessed January 13, 2021. https://www.hcup-us.ahrq.gov/toolssoftware/ccs/ccs.jsp

31. Perkins NJ, Cole SR, Harel O, et al. Principled approaches to missing data in epidemiologic studies. Am J Epidemiol. 2018;187(3):568-75.

32. Pedersen AB, Mikkelsen EM, Cronin-Fenton D, et al. Missing data and multiple imputation in clinical epidemiological research. Clin Epidemiol. 2017;9:157-66.

33. Donders AR, van der Heijden GJ, Stijnen T, Moons KG. Review: a gentle introduction to imputation of missing values. J Clin Epidemiol. 2006;59(10):1087-91.

34. van Buuren S. Multiple imputation of discrete and continuous data by fully conditional specification. Stat Methods Med Res. 2007;16(3):219-42.
35. Lee KJ, Carlin JB. Multiple imputation for missing data: fully conditional specification versus multivariate normal imputation. Am J Epidemiol. 2010;171(5):624-32.

36. Kidney Disease Improving GLobal Outcomes (KDIGO). Controverseries conference on potassium management. Miami, USA. October 2018. Accessed September 24, 2019. https://kdigo.org/ conferences/controversies-conferencepotassium-management/

37. Toto RD, Rowan CG, Alvarez P, Fogli J, Desai ND. SAT-204 patiromer and healthcare resource utilization in the real-world setting. Kidney Int Rep. 2019;4(7):S92-S93. doi: 10.1016/j.ekir.2019.05.239

38. Kidney Disease Outcomes Quality Initiative. K/DOQI clinical practice guidelines on hypertension and antihypertensive agents in chronic kidney disease. Am J Kidney Dis. 2004;43 (5 Suppl 1):S1-290. 